\title{
Práctica empresarial y desarrollo de habilidades en el aprendizaje de la Contabilidad de Gestión
}

\author{
Business practice and skills development in the learning in \\ Management Accounting
}

\author{
Pilar Ibarrondo Dávila (pdavila@ugr.es) \\ Universidad de Granada (España) \\ http://dx.doi.org/10.12795/EDUCADE.2011.i02.03
}

\begin{abstract}
RESUMEN: La interrelación entre la teoría y la práctica profesional en el ámbito de la Contabilidad de Gestión resulta necesaria y conveniente. Desde esta perspectiva, en el presente trabajo recogemos nuestra experiencia en el Proyecto de Innovación Docente titulado Docencia y Práctica Empresarial en Contabilidad de Gestión, a través del cual, hemos pretendido establecer un puente de enlace entre la docencia de la asignatura y la práctica empresarial, mediante visitas guiadas a diversas empresas de nuestro entorno, donde los alumnos han tenido la oportunidad de contrastar la aplicabilidad y utilidad práctica de los sistemas de gestión analizados en clase.
\end{abstract}

En el desarrollo del Proyecto de Innovación se ha combinado la enseñanza magistral con el desarrollo de prácticas complementarias, a través del método tutorial, la discusión dirigida y la metodología del caso. Mediante el método tutorial se ha pretendido individualizar el proceso de formación de los alumnos, en grupos reducidos. Este método se ha perfeccionado con visitas guiadas a empresas, que incluyen una charla-coloquio con sus directivos. Además, las visitas programadas a las empresas nos han permitido reconducir la discusión dirigida hacia el método de caso, acercando a los alumnos al conjunto de actividades que conlleva la toma de decisiones, situándolo en un contexto real. Entre los resultados alcanzados destacan la contribución al desarrollo de ciertas habilidades, personales e interpersonales, entre los alumnos participantes quienes, además, han mostrado un mayor interés por la adquisición de conocimientos en la asignatura, lo que se ha reflejado en sus calificaciones. Asimismo, el profesorado participante ha tenido la oportunidad de acercarse a los problemas concretos que se presentan en la práctica. Por último, resulta destacable la satisfacción alcanzada tanto por los alumnos como por los profesores que han participado en el Proyecto.

PALABRAS CLAVES: docencia y práctica empresarial, aprendizaje en contabilidad de gestión, innovación docente.

\begin{abstract}
The interrelationship between theory and practice in the field of Management Accounting is necessary and appropriate. From this perspective, this paper presents our experience in the Educational Innovation Project entitled Teaching and Business Practice in Management Accounting, through which we intend to establish a bridge between the subject teaching and business practice, through visit to the companies, where students had the opportunity to compare the applicability and usefulness, in the business practice, of the management systems discussed in class.

In this Educational Innovation Project we have combined master class with complementary practices, through the tutorial method, the discussion led and case method. Using the tutorial method we have tried to individualize the process of learning students in small groups. This method has been completed with visit to the companies. Into the results highlight the contribution made to the development of certain skills, personal and interpersonal, among pupils, who have also shown greater interest in the acquisition of knowledge in the subject, which is reflected in their grades. Also, the participating teachers had the opportunity to approach the specific problems that arise in practice.
\end{abstract}

Experiencia docente: Recibido: 24-02-11 - Versiones revisadas: 07-06-1, Aceptado: 10-09-11

Licencia Creative Commons (CC) BY-NC-ND · Asociación Española de Contabilidad y Administración de Empresas - AECA 
Finally, it is remarkable the satisfaction achieved by students and teachers involved in the Project

KEYWORDS: Teaching and business practice, Management accounting learning, Educational Innovation

\section{INTRODUCCIÓN}

La educación contable superior, como ya señalaba Beaver en 1987, resulta como consecuencia de la interrelación entre investigación, docencia y práctica empresarial. En este sentido, son numerosos los autores que ponen de manifiesto la importancia de la labor investigadora para lograr una educación de calidad.

Por otro lado, resulta evidente la necesidad de establecer vínculos entre la docencia y la práctica empresarial, al objeto de dotar a los alumnos del conocimiento necesario para el empleo de los instrumentos que posteriormente deberán aplicar en la práctica, así como para actualizar la docencia, recogiendo los avances que se hayan podido producir en los sistemas contables para la gestión de las empresas.

Por último, la relación entre la investigación y la práctica empresarial también resulta manifiesta, toda vez que en la práctica se plantean problemas que constituyen una fuente de oportunidades para la investigación, que por su parte deberá tratar de resolver dichos problemas.

Sin embargo, diversos estudios realizados en España, acerca de las interrelaciones mencionadas en el ámbito de la disciplina contablel, pusieron de manifiesto el distanciamiento existente entre la teoría y la práctica contable, lo cual resulta muy perjudicial para la docencia en Contabilidad. Asimismo, estudios más recientes a nivel internacional muestran que aún existen algunos desajustes entre docencia, investigación y práctica empresarial2.

En la docencia en Contabilidad de gestión, la interrelación entre la teoría y la práctica contable resultan convenientes y necesarias. En este sentido, de acuerdo con Blanco Dopico (2007:80-81), en los próximos años, la agenda para la investigación en Contabilidad de Gestión deberá "recurrir a una formación contable que, relacionando investigación-práctica-docencia, permita lograr un profesional con capacidades y habilidades adecuadas al actual y al futuro marco contable de gestión". Además, será necesario un "ajuste entre teoría y práctica, integrando el marco conceptual en la gestión, enlazando lo productivo y lo estratégico, esforzándose en el estudio de esa realidad, recurriendo a análisis de casos,...".

En algunas disciplinas contables el acercamiento entre teoría y práctica resulta posible sin la necesidad de establecer vínculos directos con la empresa. Tal podría ser el caso de asignaturas como Contabilidad Financiera, Análisis Contable o Auditoría, en las que, al disponer de información económico financiera pública de las empresas, ésta resulta accesible para los profesores y alumnos, a través de bases de datos privadas existentes en el mercado o, incluso, a través del propio Registro Mercantil, donde las empresas tienen la obligación de depositar sus Cuentas Anuales.

\footnotetext{
1 Puede consultarse al respecto: García Benau et al. (1996); García Benau (1997); Tua (1992); Blanco Dopico y Aibar, (2001); y Escobar y Lobo (2000), entre otros.

2 Puede consultarse al respecto: Cable et al. (2009); Baldvinsdottir et al. (2010); y Tan et al. (2004), entre otros.
} 
En cambio, las asignaturas de Contabilidad de Costes y de Gestión, al ocuparse de sistemas de información gestionados por, y para, usuarios internos de las empresas, no disponen de una base de datos real accesible para lograr el necesario vínculo entre la teoría y la práctica empresarial. Tan sólo se pueden consultar, en el mejor de los casos, las experiencias realizadas por ciertos investigadores y publicadas en forma de estudios de caso.

Si bien es cierto que la utilidad del método de caso como herramienta didáctica, según destacan Arquero y Jiménez (1999), ha sido respaldada por organizaciones contables, como IFAC ${ }^{3}$ (1994 y 1996) o AAA (1986), y por algunas firmas de auditoría, como Arthur Andersen \& Co. (1989), a nuestro juicio, el mejor camino posible para conciliar teoría y práctica en la docencia de la Contabilidad de Costes y de Gestión debería ser a través del conocimiento directo de las prácticas contables internas aplicadas en las empresas, que sería posible, básicamente, por dos vías: mediante charlas de profesionales vinculados al mundo de la empresa, o a través de visitas guiadas a empresas del entorno.

Si bien la primera de las alternativas puede ofrecer una visión general de los sistemas de gestión aplicados en las empresas, creemos que sólo la segunda puede ofrecer una visión concreta sobre los temas específicos abordados en la asignatura, sin perjuicio de la perspectiva global, puesto que la visita a las instalaciones de las empresas se puede combinar con charlas-coloquio en el seno de las mismas entre los profesionales, los alumnos y los profesores. Además, la visita a las instalaciones productivas de las empresas es la mejor forma de adquirir un conocimiento de primera mano de los procesos de transformación, lo que posibilita la comprensión de las relaciones causa-efecto que se desprenden de dichos procesos, así como la aplicación de los distintos sistemas de cálculo de costes. Esta última vía ha sido la que ha presidido el desarrollo de nuestro proyecto de innovación docente.

Por otro lado, el enfoque seguido por el Espacio Europeo de Educación Superior para el proceso de enseñanza-aprendizaje, ha sido el otro gran eje sobre el que ha girado el desarrollo de nuestro proyecto de innovación docente. En efecto, dicho enfoque pone de relieve la importancia que presenta el fomento de las capacidades y destrezas que se requieren para el aprendizaje a lo largo de toda la vida, así como de aquellas habilidades y competencias que capacitarán al estudiante para el ejercicio de la profesión correspondiente. Por ello, tomando como referencia los pronunciamientos de organismos contables internacionales, tales como AECC (1990), AICPA (1988, 1992 y 1999) O IFAC (1994 y 1996), y de acuerdo con Arquero y Donoso (1998), la educación contable superior debe proporcionar individuos preparados para la adaptación al cambio, con una base formativa de conocimientos, valores profesionales y capacidades personales, destacando entre estas últimas, las de comunicación, las interpersonales o de grupo y las intelectuales.

\section{OBJETIVOS DEL PROYECTO}

En las líneas de introducción precedentes, hemos puesto de relieve la importancia que presenta el establecimiento de vínculos entre la docencia, la investigación y la práctica contable empresarial, así como la necesidad de proporcionar a los alumnos habilidades y destrezas que les capaciten para su formación continua y para el ejercicio de su actividad profesional. Con el ánimo de contribuir al logro de dichos fines generales, nos fijamos los siguientes objetivos en el desarrollo de nuestro proyecto de innovación docente:

3 International Federation of Accountants, American Accounting Association, Accounting Education Change Commission, American Institute of Certified Public Accountants. 
1.- Fomentar la interrelación entre profesores, alumnos y profesionales (directivos, gestores de información contable, ...).

2.- Mejorar el conocimiento de profesores y alumnos mediante el acercamiento a la utilidad práctica de los temas abordados en clase.

3.- Procurar un acercamiento entre los problemas que se producen en la práctica contable empresarial y las soluciones que se proponen desde la teoría contable.

4.- Contribuir al desarrollo de habilidades personales e interpersonales en los alumnos, mediante trabajos de grupo, presentación en clase, interacción con los profesionales de las empresas visitadas, debates, ...

5.- Lograr una mayor individualización del proceso de formación intelectual de los alumnos mediante el método tutorial.

6.- Contribuir a generar un espíritu crítico y creativo en los alumnos, mediante la discusión dirigida en torno a los temas abordados en los trabajos, tanto en clase como en las visitas a las empresas.

7.- Acercar a los alumnos a la investigación, mediante la tutela de un trabajo escrito centrado en el análisis de temáticas concretas, lo que les obliga a familiarizarse con el uso de bases de datos específicas y a efectuar revisiones bibliográficas.

8.- Mejorar la destreza de los alumnos con las tecnologías de la información y comunicación, mediante la utilización de los medios informáticos necesarios para su elaboración y presentación.

9.- Elaborar material didáctico audiovisual, que pueda ser de utilidad para el desarrollo de cursos posteriores, con las grabaciones de las visitas realizadas a las empresas.

\section{DESCRIPCIÓN DE LA EXPERIENCIA}

Durante el curso 2006-07 realizamos una experiencia previa a la solicitud del Proyecto de Innovación Docente en la Universidad de Granada. Esta experiencia piloto nos ofreció la oportunidad de diseñar el Proyecto conociendo las ventajas y las limitaciones que el mismo podía presentar, y nos puso de manifiesto la necesidad de contar con recursos que sólo a través de la concesión de un Proyecto de Innovación Docente sería posible obtener.

El número de alumnos matriculados en las asignaturas de Contabilidad de Costes y de Gestión 4 fue la primera dificultad que nos encontramos a la hora de llevar a cabo el Proyecto. Por esta razón, decidimos implantarlo inicialmente en una asignatura optativa, Contabilidad de Gestión, en la que anualmente se matriculan en torno a 200 alumnos. Aún así, las visitas a empresas nos exigían restringir el número de alumnos, por lo que decidimos hacerlo a través de prácticas voluntarias, consistentes en la realización de un trabajo en grupo sobre temas específicos abordados en la asignatura, que culmina con su exposición pública en clase. Además, la visita a las empresas nos ha permitido organizar debates dirigidos con los directivos de dichas empresas, en torno a los temas analizados en los trabajos realizados por los alumnos, mediante la formulación de preguntas por parte de los propios estudiantes, previamente filtrados por las profesoras.

\footnotetext{
${ }^{4}$ Más de 1000 alumnos matriculados en cada curso académico, al considerar el agregado global de todas las titulaciones.
} 
La experiencia se ha repetido en los cursos académicos 07-08 y 08-09, llegando en todos los casos a resultados muy similares.

El desarrollo de las prácticas voluntarias ha tenido lugar en las siguientes etapas:

1.- Planificación de las prácticas

2.- Tutela de los trabajos en grupo.

3.- Visita a las empresas

4.- Exposición de los trabajos en clase

5.- Elaboración de material didáctico audiovisual.

\subsection{PLANIFICACIÓn de LAS PRÁCticas.}

En mayo de 2006 se iniciaron los contactos con las empresas en las que posteriormente se realizaron las visitas (HEFAGRA, DEGRÁ, PULEVA y DHUL), todas ellas empresas líderes en el sector económico al que pertenecen. La propuesta fue recibida con entusiasmo por la dirección de todas ellas, sensibles a la necesidad de una más estrecha colaboración entre empresa y universidad, para mejorar la docencia que forma a los futuros profesionales de la gestión empresarial, máxime en nuestra área de conocimiento, por los motivos que indicamos en la introducción. Los directivos de las empresas indicadas y el profesorado adscrito al Proyecto coincidimos, desde el primer momento, en los beneficios que se derivarían para el alumnado participante.

En cada uno de los cursos académicos en los que se desarrolló el proyecto, durante el mes de octubre se realizó la planificación global de las prácticas relativas al proyecto presentado, mediante la difusión de la metodología a seguir entre los alumnos de la asignatura, la recogida de las solicitudes de participación en un impreso diseñado para tal efecto, la formación de los grupos de trabajo y la asignación a cada grupo del tema objeto del trabajo; temas que, preferentemente, coincidían con los desarrollos más recientes en Contabilidad de Gestión.

Paralelamente se concretaron las fechas en que podrían celebrarse las visitas a las empresas. En la experiencia piloto de 2006/07 la visita se realizó a HEFAGRA, en el curso 2007/08 se visitó HEFAGRA y PULEVA y, finalmente, en 2008/09 se visitó HEFAGRA y DHUL. DEGRÁ participó sólo en la experiencia preliminar, porque las responsables del Proyecto consideramos oportuno centrarnos en aquellas empresas que nos ofrecían mayor potencial para la docencia en nuestra asignatura. Por esta misma razón, la visita a HEFAGRA aparece de forma recurrente en todos los cursos, en tanto que constituye un referente nacional en tecnología robótica para la distribución farmacéutica y utiliza sistemas avanzados de información para la gestión.

\subsection{TUTELA DE LOS TRABAJOS EN GRUPO}

Durante el mes de noviembre, de cada edición, se realizaron las primeras rondas de reuniones entre el profesor-tutor y los alumnos, con el objetivo de orientarlos inicialmente en la elaboración del trabajo (estructura preliminar de los contenidos a tratar, búsquedas bibliográficas y manejo de bases de datos electrónicas).

En los meses de diciembre y primeros de enero, tuvieron lugar las segundas rondas de reuniones de tutorización, al objeto de orientar a los alumnos en el desarrollo y presentación del trabajo (revisión final de contenidos, preparación conjunta de las cuestiones que iban a plantear a los directivos que nos atendieran en las visitas, posterior intercambio de impresiones acerca de las mismas, orientación acerca de cómo incluir dicha experiencia práctica en el cuerpo del documento cuya redacción 
se encontraban culminando, orientación para la presentación formal del informe escrito, transmitiéndoles la importancia de una cuidada redacción -con especial referencia a la introducción y las conclusiones- así como la manera adecuada de referenciar las citas bibliográficas).

A mediados de enero se efectuó la tercera ronda de reuniones entre el profesor-tutor y los alumnos en la que se entregó el trabajo escrito elaborado por cada grupo y se orientó para la exposición pública del mismo, utilizando para ello el programa informático power point.

\subsection{VISITA A LAS EMPRESAS}

Durante el curso 2006-07 se realizó una visita a HEFAGRA, en sus antiguas instalaciones, concretamente, en enero de 07. En estas fechas, la empresa estaba llevando a cabo un proceso de modernización, teniendo lugar en los siguientes meses su traslado a las nuevas instalaciones, totalmente automatizadas. Esta circunstancia nos brindó la oportunidad de obtener imágenes y vídeos de sus antiguas instalaciones, para poder mostrarlas a los alumnos que visitaran las nuevas instalaciones en los siguientes cursos.

En el curso siguiente, durante el mes de diciembre de 2007 tuvo lugar la visita a las nuevas instalaciones de la empresa HEFAGRA, con una parte de los grupos de trabajo. En esta empresa nos permitían llevar un mayor número de alumnos por lo que decidimos realizar la visita con los alumnos de prácticas del grupo de mañana, al ser éste el más numeroso.

La visita a la empresa se inició con la recepción que nos hicieron D. Jesús Porres Ureña, Director General, y D. José Luis García del Rosal, Director de Producción y Operaciones. En dicha recepción, el Director General nos ofreció una conferencia para explicarnos el funcionamiento de las instalaciones industriales, que posteriormente visitaríamos, así como los sistemas de gestión empleados en la empresa para el cálculo de costes, la gestión de la calidad, la gestión medioambiental, el cuadro de mando integral y la cadena de valor de la empresa. La conferencia finalizó en un coloquio en el que los alumnos tuvieron la oportunidad de efectuar preguntas relacionadas con el tema del trabajo que estaban elaborando. A continuación, el Director de Producción y Operaciones nos acompañó en la visita guiada a las instalaciones. Allí, nos explicó el funcionamiento de la cadena de producción, que acababa de ser inaugurada, y que, según nos indicó, se había convertido en la instalación de distribución farmacéutica más moderna de Europa.

En enero de 2008, tuvo lugar la visita a la empresa PULEVA con el resto de los grupos de prácticas. En esta ocasión, la visita comenzó con el reconocimiento de las instalaciones productivas donde dos técnicos industriales nos fueron explicando su funcionamiento. Posteriormente, D. Gabriel Martín Ocaña, Subdirector GeneralAdministración y Finanzas, D. Javier Cano Dard, Responsable de Control de Gestión, y D. César Cenarro, Responsable de la Información Económico-Financiera, nos ofrecieron una charla-coloquio en la que nos mostraron y explicaron la elaboración de sus informes financieros y de gestión, el proceso de cálculo de costes, el cuadro de mando integral y su sistema de gestión de calidad. Al finalizar su exposición, los alumnos formularon diversas cuestiones en relación a los temas tratados en la visita y abordados en sus trabajos.

Finalmente, durante el curso 08-09, se reeditó la visita a HEFAGRA con los nuevos alumnos, en los términos comentados para el curso anterior. Además, en enero de 09, se realizó una visita a la empresa DHUL, en la que su Director técnico, D. Antonio Marín, nos ofreció un charla-coloquio sobre la gestión del proceso productivo y los informes 
que se elaboran para los distintos niveles de responsabilidad. Al final de la exposición nos mostraron sus instalaciones productivas.

\subsection{EXPOSICIÓN DE LOS TRABAJOS EN CLASE}

En la última semana lectiva del cuatrimestre se expusieron los trabajos en clase ante el resto de los compañeros, siendo obligatoria la participación de todos los miembros del grupo en dicha exposición. En las exposiciones públicas el profesor-tutor trató de fomentar el debate crítico entre los alumnos mediante el método de la discusión dirigida.

\subsection{ELABORACIÓN DE MATERIAL DIDÁCTICO AUDIOVISUAL.}

En la visita realizada a la empresa HEFAGRA nos permitieron grabar las instalaciones técnicas, lo que posteriormente nos sirvió para elaborar material didáctico audiovisual, que será de utilidad para los próximos cursos académicos. En la visita a PULEVA y a DHUL, por motivos de seguridad, no estaba permitido realizar grabaciones, no obstante, en PULEVA los directivos nos facilitaron material audiovisual que también nos será de gran utilidad para los próximos cursos.

\section{METODOLOGÍA APLICADA}

Siguiendo las directrices que marca el Espacio Europeo de Educación Superior, las prácticas desarrolladas resultan complementarias con la enseñanza magistral a través del método tutorial, la discusión dirigida y la metodología del caso.

Con el método tutorial se pretende individualizar el proceso de formación intelectual de los alumnos. En nuestro caso, se ha llevado a cabo en varias reuniones programadas con cada uno de los grupos de trabajo, formados, cada uno de ellos, por tres alumnos. La labor prioritaria del profesor-tutor ha sido la de desarrollar las capacidades de sus alumnos tutelados mediante una orientación intelectual personalizada. En tales sesiones, más que proporcionar información, el profesor ha procurado enseñar a pensar y a argumentar al alumno, a partir del trabajo que cada grupo debía elaborar. La crítica del tutor persigue que el alumno argumente y defienda sus posiciones, que contemple nuevos puntos de vista $y$, en definitiva, le conduzca hacia una mayor profundización en la materia. El método tutorial, así entendido, se complementa con la lectura de otros trabajos relacionados, bajo las directrices del tutor; con las lecciones magistrales; $y$, se perfecciona, en nuestra experiencia concreta, con la asistencia a la visita guiada a una empresa, que incluye una charla-coloquio con sus directivos, en la que se abordan los temas sobre los que los alumnos han desarrollado sus trabajos.

La discusión dirigida consiste en la deliberación de un grupo de alumnos acerca de un tema determinado bajo la coordinación del profesor. Su aplicación requiere que la cuestión objeto de debate admita distintos puntos de vista a partir de los cuales pueda suscitarse la discusión y presupone el dominio de los conocimientos necesarios acerca del tema por parte del alumno.

Finalmente, las visitas programadas a las empresas nos han permitido reconducir la discusión dirigida hacia el método del caso, enfrentando a los alumnos con el conjunto de actividades que conlleva la toma de decisiones, situándolo en un contexto real. Es un método eminentemente orientado hacia la práctica profesional que pretende estimular una serie de facetas personales como la comunicación oral y escrita, la interacción con el grupo, la capacidad para solventar problemas, tratar con situaciones ambiguas, comprender mejor el mundo real, etc. No pretende incrementar 
los conocimientos del alumno, sino permitirle aplicar aquellos que ha adquirido por otros métodos.

Por otro lado, en el desarrollo de las prácticas voluntarias, se ha promovido la aplicación de las tecnologías de la información. Los alumnos han empleado los actuales medios informáticos, tanto para la redacción del informe escrito, como para la búsqueda bibliográfica y la exposición pública del trabajo.

Finalmente, la elaboración de vídeos didácticos a partir de las visitas realizadas a las empresas puede resultar muy interesante para ayudar al estudiante a conocer la problemática real de las empresas. En este sentido, a través del proyecto que hemos llevado a cabo hemos podido filmar y fotografiar digitalmente el desarrollo de las visitas guiadas, así como los procesos productivos de las empresas colaboradoras, lo que nos ha permitido elaborar y editar material didáctico innovador que podrá ser utilizado en la docencia de las asignaturas afectadas en futuros cursos académicos.

\section{GRADO DE SATISFACCIÓN DEL ALUMNADO PARTICIPANTE}

La metodología utilizada para la evaluación del Proyecto ha sido la elaboración de encuestas a todos los alumnos participantes en el mismo, en las experiencias llevadas a cabo durante los cursos 06-07, 07-08 y 08-09.

\subsection{ENCUESTA CURSO 06-07}

En la encuesta realizada a los alumnos participantes en las prácticas voluntarias durante el curso 2006-07, se puso de manifiesto que entre los objetivos que les habían motivado a participar en las prácticas referidas, según se desprende de la Tabla 1, destaca, en primer lugar, el logro de una mayor puntuación en la nota final, objetivo para el que más del 87 por 100 de los alumnos otorgan la valoración de bastante-muy importante; seguido del interés por conocer la aplicación de las herramientas de gestión en una empresa real, objetivo para el que cerca del 70 por 100 de los alumnos asignan dicha valoración; a continuación le sigue el objetivo de profundización en el conocimiento de los temas abordados en la asignatura $(66,66$ por 100) y, por último, el desarrollo de habilidades de trabajo autónomo y autoaprendizaje (60,60 por 100).

TABLA 1. IMPORTANCIA DE LOS OBJETIVOS PERSEGUIDOS EN LA PARTICIPACIÓN DE LOS ALUMNOS. CURSO 06-07.

\begin{tabular}{|c|c|c|c|c|c|c|c|c|c|c|c|c|}
\hline & \multicolumn{2}{|c|}{$\begin{array}{c}\text { Nada } \\
\text { importante }\end{array}$} & \multicolumn{2}{|c|}{$\begin{array}{c}\text { Poco } \\
\text { importante }\end{array}$} & \multicolumn{2}{|c|}{$\begin{array}{l}\text { Nivel medio } \\
\text { importancia }\end{array}$} & \multicolumn{2}{|c|}{$\begin{array}{c}\text { Bastante } \\
\text { importante }\end{array}$} & \multicolumn{2}{|c|}{$\begin{array}{c}\text { Muy } \\
\text { importante }\end{array}$} & \multicolumn{2}{|c|}{ Total } \\
\hline & Frec. & $\%$ & Frec. & $\%$ & Frec. & $\%$ & Frec. & $\%$ & Frec. & $\%$ & Frec. & $\%$ \\
\hline $\begin{array}{l}\text { Profundizar en el conocimiento de } \\
\text { los temas abordados en la } \\
\text { asignatura }\end{array}$ & & & 5 & 15,15 & 6 & 18.18 & 10 & 30,30 & 12 & 36,36 & 33 & 100.00 \\
\hline $\begin{array}{l}\text { Desarrollar habilidades de trabajo } \\
\text { autónomo y autoaprendizaje } \\
\text { Alcanzar una mayor puntuación en }\end{array}$ & 1 & 3,03 & 4 & 12,12 & 8 & 24,24 & 13 & 39,39 & 7 & 21,21 & 33 & 100,00 \\
\hline $\begin{array}{l}\text { la nota final } \\
\text { Conocer la aplicación en una } \\
\text { empresa real de las herramientas } \\
\text { de gestión }\end{array}$ & 1 & 3,23 & 1 & 3.03 & 3 & 9,68 & 11 & 35,48 & 16 & 51,61 & 31 & 100,00 \\
\hline
\end{tabular}

Otro grupo de ítems de la encuesta indaga sobre la influencia, percibida por los alumnos, en la mejora de ciertas habilidades personales. De acuerdo con los datos recogidos en la Tabla 2, nos llama la atención el hecho de que un elevado porcentaje de alumnos, el 81,81 por 100, ha percibido como bastante o muy positiva la influencia de las prácticas en el nivel de conocimiento de los temas abordados, teniendo en cuenta que esta cuestión presentaba una menor relevancia entre los objetivos que los 
alumnos perseguían con su participación en las prácticas. Además, el 63,63 por 100 le ha otorgado dicha valoración al desarrollo de habilidades de trabajo en equipo y más del 70 por 100 de ellos ha considerado bastante o muy positiva la influencia en su capacidad de comunicación y en su destreza en la utilización de las nuevas tecnologías, por lo que se pone de manifiesto un elevado grado de consecución en los objetivos que nos habíamos propuesto con el desarrollo del Proyecto.

TABLA 2. INFLUENCIA DE LA REALIZACIÓN DEL TRABAJO DE PRÁCTICAS EN LAS HABILIDADES PERSONALES DE LOS ALUMNOS. CURSO 06-07.

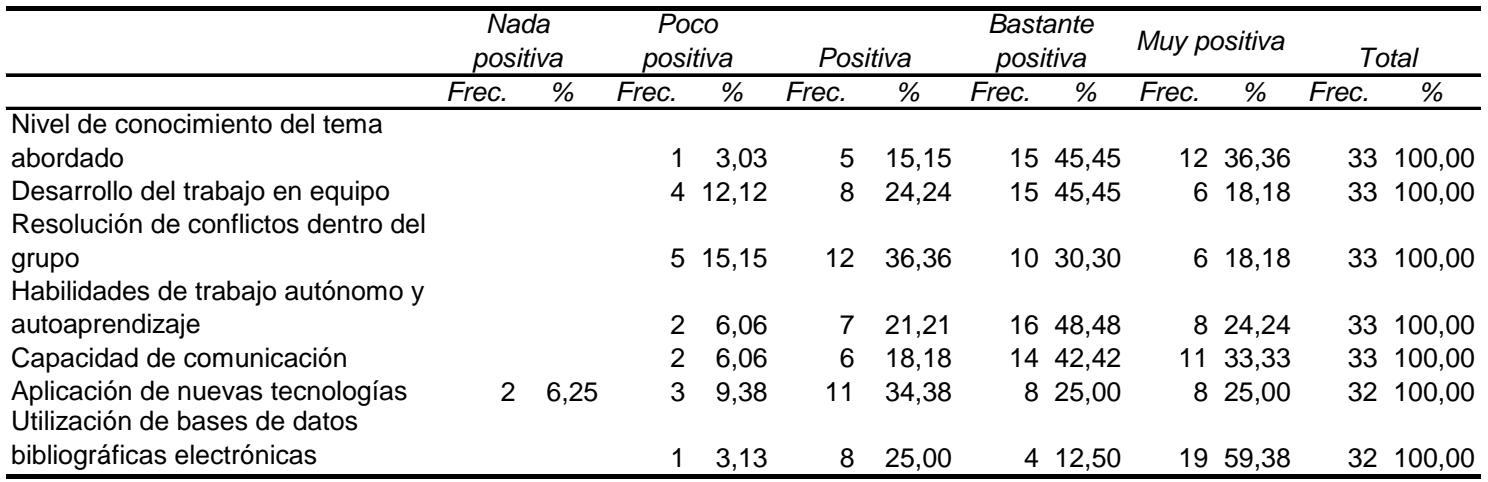

Durante el curso 06-07, entre los alumnos que participaron en la práctica sólo el 57,58 por 100 realizaron la visita a la empresa. Entre éstos últimos el nivel de satisfacción se mostró mucho más elevado que en el resto de los alumnos participantes. Por esta razón decidimos que, en el desarrollo de trabajos en cursos posteriores, la visita a la empresa sería obligatoria para todos los alumnos participantes.

En efecto, como muestra la Tabla 3, entre los alumnos que realizaron la visita a la empresa, cerca del 90 por 100 estimaron bastante o muy positivo el acercamiento a la práctica empresarial en los temas tratados; y más del 70 por 100 de los alumnos otorgaron dicha valoración al nivel de conocimientos alcanzados en los temas trabajados, lo que nuevamente pone de manifiesto el elevado grado de consecución de los objetivos que perseguíamos con el desarrollo del Proyecto.

TABLA 3. INFLUENCIA DE LAS VISITAS A LAS EMPRESAS EN LAS HABILIDADES PERSONALES DE LOS ALUMNOS. CURSO 06-07.

\begin{tabular}{|c|c|c|c|c|c|c|c|c|c|c|c|c|}
\hline & \multicolumn{2}{|c|}{$\begin{array}{c}\text { Nada } \\
\text { positiva }\end{array}$} & \multicolumn{2}{|c|}{$\begin{array}{l}\text { Poco } \\
\text { positiva }\end{array}$} & \multicolumn{2}{|c|}{ Positiva } & \multicolumn{2}{|c|}{$\begin{array}{l}\text { Bastante } \\
\text { positiva }\end{array}$} & \multicolumn{2}{|c|}{ Muy positiva } & \multicolumn{2}{|c|}{ Total } \\
\hline & Frec. & $\%$ & Frec. & $\%$ & Frec. & $\%$ & Frec. & $\%$ & Frec. & $\%$ & Frec. & $\%$ \\
\hline $\begin{array}{l}\text { Acercamiento a la práctica } \\
\text { empresarial en los temas tratados }\end{array}$ & & & & & & & & & & & & \\
\hline en la asignatura & & & & & 2 & 10,53 & 8 & 42,11 & 9 & 47,37 & 19 & 100,00 \\
\hline $\begin{array}{l}\text { Nivel de conocimiento de los } \\
\text { temas abordados }\end{array}$ & & & & & 5 & 26,32 & 9 & 47,37 & 5 & 26,32 & 19 & 100,00 \\
\hline Capacidad de comunicación & & & 1 & 5,26 & 6 & 31,58 & 9 & 47,37 & 3 & 15,79 & 19 & 100,00 \\
\hline Aplicación de nuevas tecnologías & 1 & 5,26 & & & 6 & 31,58 & 8 & 42,11 & 4 & 21,05 & 19 & 100,00 \\
\hline
\end{tabular}

Por último, los resultados de la encuesta realizada a los alumnos participantes durante el curso 06-07 muestran una elevada satisfacción, teniendo en cuenta que la valoración global de la utilidad que les había reportado las prácticas voluntarias en Contabilidad de Gestión alcanzaba una puntuación media 4,15 sobre 5.

\subsection{ENCUESTA CURSO 07-08}

En la encuesta realizada entre los alumnos participantes durante el curso 07-08, como muestra la Tabla 4, nuevamente resultan destacables como principales objetivos en su 
participación el obtener una mayor puntuación en la nota final, objetivo al que más del 90 por 100 le otorga la valoración de bastante o muy importante, seguido del objetivo de conocer la aplicación de los instrumentos de gestión en una empresa real, que recibe esa valoración por más del 77 por 100.

TABLA 4. IMPORTANCIA DE LOS OBJETIVOS PERSEGUIDOS EN LA PARTICIPACIÓN DE LOS ALUMNOS.CURSO 07-08.

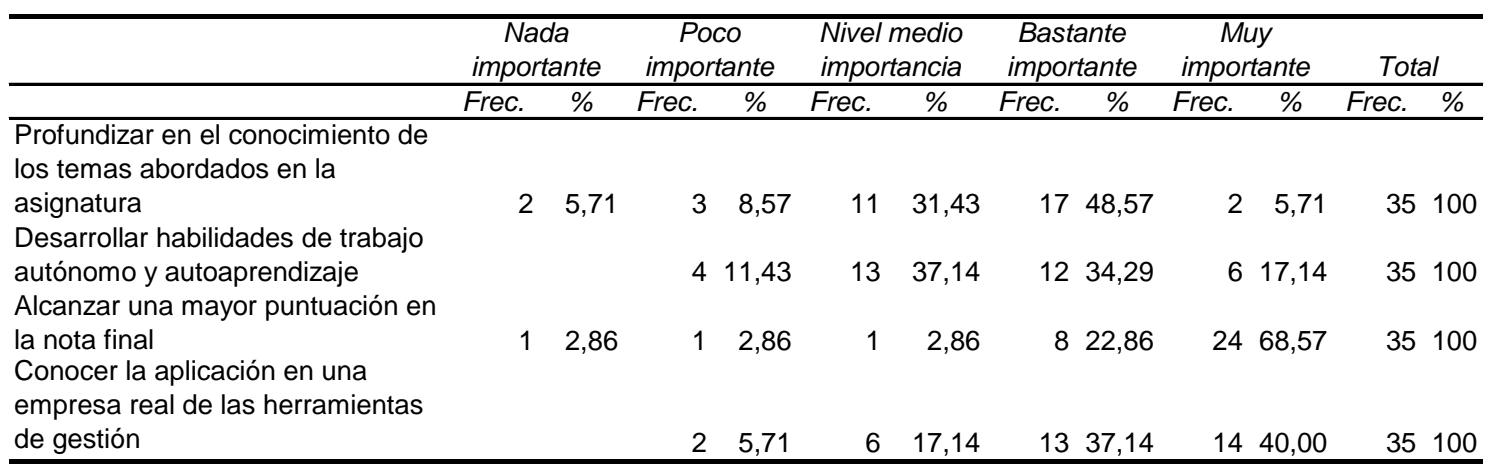

La Tabla 5 recoge la valoración de la influencia ejercida, según la percepción de los propios alumnos, por la elaboración de los trabajos en ciertas habilidades personales, donde puede observarse que destaca, en primer lugar, el desarrollo del trabajo en equipo, seguido del nivel de conocimientos alcanzados en relación a los temas abordados $^{5}$, de la resolución de conflictos dentro del grupo y la destreza en la utilización de las nuevas tecnologías, a los que más del 70 por 100 de los alumnos le otorgan la calificación de bastante o muy positiva, por lo que nuevamente se pone de relieve el alto grado de consecución de los objetivos que nos habíamos propuesto con el desarrollo del Proyecto.

TABLA 5. INFLUENCIA DE LA REALIZACIÓN DEL TRABAJO DE PRÁCTICAS EN LAS HABILIDADES PERSONALES DE LOS ALUMNOS. CURSO 07-08.

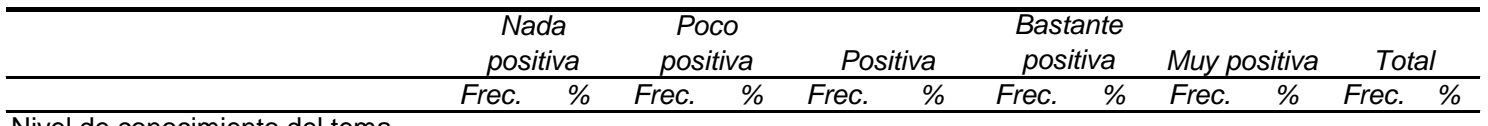

Nivel de conocimiento del tema abordado

Desarrollo del trabajo en equipo

Resolución de conflictos dentro del grupo

$\begin{array}{lllllllll}9 & 28,13 & 11 & 34,38 & 12 & 37,50 & 32 & 100\end{array}$

Habilidades de trabajo autónomo y

autoaprendizaje

Capacidad de comunicación

Aplicación de nuevas tecnologías

Utilización de bases de datos

$\begin{array}{llllllllll}2 & 5,71 & 1 & 2,86 & 6 & 17,14 & 16 & 45,71 & 10 & 28,57\end{array}$

bibliográficas electrónicas

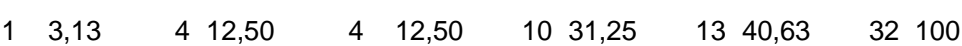

En relación a la valoración de la influencia que hayan podido tener las actividades desarrolladas en las visitas guiadas a las empresas, en la mejora de ciertas capacidades personales de los alumnos, destaca nuevamente el acercamiento a la

\footnotetext{
${ }^{5}$ Al igual que en desarrollo de las prácticas durante el curso 2006-07, en el curso 2007-08 nos llama la atención que, si bien, tan sólo el $54,28 \%$ de los alumnos valoraban como bastante o muy importante, en su participación en las prácticas voluntarias, el objetivo de profundizar en el conocimiento de los temas abordados en clase, sin embargo, el $71,88 \%$ reconoce que la influencia de su participación en las prácticas ha sido bastante o muy positiva en la adquisición de conocimientos en los temas tratados, lo cual nos parece un logro muy relevante en la consecución de los objetivos que nos habíamos establecido con el desarrollo del Proyecto.
} 
práctica empresarial de los temas abordados en sus trabajos6, seguida del nivel de conocimiento alcanzado en dichas temáticas ${ }^{7}$, como se observa en la Tabla 6.

TABLA 6. INFLUENCIA DE LAS VISITAS A LAS EMPRESAS EN LAS HABILIDADES PERSONALES DE LOS ALUMNOS. CURSO 07-08.

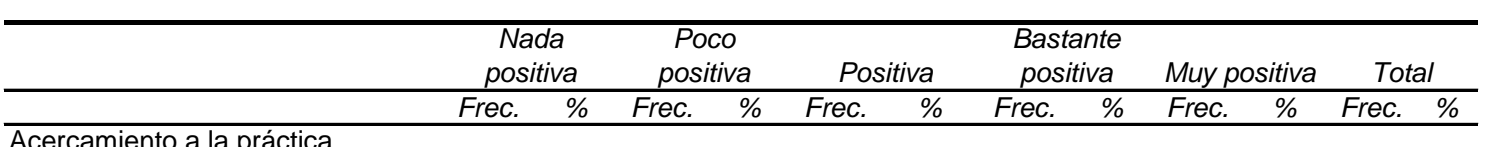

Acercamiento a la práctica

empresarial en los temas tratados

en la asignatura

abordados

Capacidad de comunicación

13,13

$2 \quad 6,25 \quad 3 \quad 9,38$

928,13

1753,13

32100

$\begin{array}{rrrrr}1 & 2 & 6,25 & 6 & 18,75\end{array}$

1650,00

825,00

32100

Aplicación de nuevas tecnologías

$\begin{array}{llllll}2 & 6,25 & 2 & 6,25 & 8 & 25,00\end{array}$

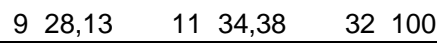

Por último, nuevamente se observa una gran satisfacción del alumnado en su participación en las prácticas voluntarias, según se refleja en la valoración global que los alumnos conceden a la utilidad de las prácticas realizadas, que se sitúa en 4,11 sobre 5 puntos.

\subsection{ENCUESTA CURSO 08-09}

Finalmente, en base a los resultados obtenidos de la encuesta llevada a cabo entre los alumnos participantes durante el curso 08-09, resulta nuevamente destacable como principales objetivos en su participación el obtener una mayor puntuación en la nota final, objetivo al que más del 96 por 100 le otorga la valoración de bastante o muy importante, seguido del objetivo de conocer la aplicación de los instrumentos de gestión en una empresa real, que recibe esa valoración por más del 73 por 100, como se refleja en la Tabla 7.

TABLA 7. IMPORTANCIA DE LOS OBJETIVOS PERSEGUIDOS EN LA PARTICIPACIÓN DE LOS ALUMNOS. CURSO 08-09

\begin{tabular}{|c|c|c|c|c|c|c|c|c|c|c|c|c|}
\hline & \multicolumn{2}{|c|}{$\begin{array}{l}\text { Nada } \\
\text { importante }\end{array}$} & \multicolumn{2}{|c|}{$\begin{array}{c}\text { Poco } \\
\text { importante }\end{array}$} & \multicolumn{2}{|c|}{$\begin{array}{l}\text { Nivel medio de } \\
\text { importancia }\end{array}$} & \multicolumn{2}{|c|}{$\begin{array}{l}\text { Bastante } \\
\text { importante }\end{array}$} & \multicolumn{2}{|c|}{$\begin{array}{l}\text { Muy } \\
\text { importante }\end{array}$} & \multicolumn{2}{|c|}{ Total } \\
\hline & Frec. & $\%$ & Frec. & $\%$ & Frec. & $\%$ & Frec. & $\%$ & Frec. & $\%$ & Frec. & $\%$ \\
\hline $\begin{array}{l}\text { Profundizar en el conocimiento de los temas } \\
\text { abordados en la asignatura }\end{array}$ & 2 & 3,51 & 2 & 3,51 & 27 & 47,37 & 20 & 35,09 & 6 & 10,53 & 57 & 100 \\
\hline $\begin{array}{l}\text { Desarrollar habilidades de trabajo autónomo } \\
\text { y autoaprendizaje }\end{array}$ & 1 & 1,75 & 6 & 10,53 & 17 & 29,82 & 24 & 42,11 & 9 & 15,79 & 57 & 100 \\
\hline $\begin{array}{l}\text { Alcanzar una mayor puntuación en la nota } \\
\text { final }\end{array}$ & & & 1 & 1,75 & 1 & 1,75 & 8 & 14,04 & 47 & 82,46 & 57 & 100 \\
\hline $\begin{array}{l}\text { Conocer la aplicación en una empresa real } \\
\text { de las herramientas de gestión }\end{array}$ & & & 7 & 12,28 & 8 & 14,04 & 32 & 56,14 & 10 & 17,54 & 57 & 100 \\
\hline
\end{tabular}

En la valoración de la influencia ejercida por la elaboración de los trabajos en ciertas habilidades personales, si analizamos el porcentaje de alumnos que les otorgan la calificación de bastante o muy positiva, destaca, en primer lugar, la capacidad de comunicación $(78,9 \%)$, el trabajo autónomo $(75,4 \%)$, el aprendizaje en la utilización de

\footnotetext{
${ }^{6}$ El $81,26 \%$ de los alumnos estima que la visita realizada a la empresa ha ejercido una influencia bastante o muy positiva en el acercamiento a la práctica empresarial en los temas tratados en la asignatura.

${ }^{7}$ El $75 \%$ de los alumnos considera que la visita a la empresa ha ejercido una influencia bastante o muy positiva en el nivel de conocimientos adquiridos sobre los temas abordados.
} 
bases de datos bibliográficas electrónicas $(70,2 \%)$, el nivel de conocimiento del tema abordado $(69,2 \%)^{8}$ y el desarrollo del trabajo en equipo $(66,7 \%)$, según se muestra en la Tabla 8.

TABLA 8. INFLUENCIA DE LA REALIZACIÓN DEL TRABAJO DE PRÁCTICAS EN LAS HABILIDADES PERSONALES DE LOS ALUMNOS Y EN SU NOTA FINAL. CURSO 08-09

\begin{tabular}{|c|c|c|c|c|c|c|c|c|c|c|c|c|}
\hline & \multicolumn{2}{|c|}{$\begin{array}{c}\text { Nada } \\
\text { positiva }\end{array}$} & \multicolumn{2}{|c|}{ Poco positiva } & \multicolumn{2}{|c|}{ Positiva } & \multicolumn{2}{|c|}{$\begin{array}{l}\text { Bastante } \\
\text { positiva }\end{array}$} & \multicolumn{2}{|c|}{ Muy positiva } & \multicolumn{2}{|c|}{ Total } \\
\hline & Frec. & $\%$ & Frec. & $\%$ & Frec. & $\%$ & Frec. & $\%$ & Frec. & $\%$ & Frec. & $\%$ \\
\hline Nivel de conocimiento del tema abordado & & & 1 & 1,79 & 6 & 10,71 & 29 & 51,79 & 20 & 35,71 & 56 & 100 \\
\hline Desarrollo del trabajo en equipo & & & 2 & 3,51 & 17 & 29,82 & 25 & 43,86 & 13 & 22,81 & 57 & 100 \\
\hline Resolución de conflictos dentro del grupo & 3 & 5,26 & 10 & 17,54 & 17 & 29,82 & 16 & 28,07 & 11 & 19,30 & 57 & 100 \\
\hline $\begin{array}{l}\text { Habilidades de trabajo autónomo y } \\
\text { autoaprendizaje }\end{array}$ & & & 4 & 7,02 & 10 & 17,54 & 35 & 61,40 & 8 & 14,04 & 57 & 100 \\
\hline Capacidad de comunicación & 1 & 1,75 & 1 & 1,75 & 10 & 17,54 & 26 & 45,61 & 19 & 33,33 & 57 & 100 \\
\hline Aplicación de nuevas tecnologías & 2 & 3,51 & 4 & 7,02 & 23 & 40,35 & 13 & 22,81 & 15 & 26,32 & 57 & 100 \\
\hline $\begin{array}{l}\text { Utilización de bases de datos bibliográficas } \\
\text { electrónicas }\end{array}$ & 1 & 1,75 & 7 & 12,28 & 9 & 15,79 & 27 & 47,37 & 13 & 22,81 & 57 & 100 \\
\hline Nota final & & & 3 & 6,12 & 1 & 2,04 & 19 & 38,78 & 26 & 53,06 & 49 & 100 \\
\hline
\end{tabular}

En la encuesta realizada a los alumnos participantes en el curso 2008-09 se ha incluido un nuevo ítem, para indagar sobre el grado de satisfacción del alumnado en la repercusión de su participación en las prácticas voluntarias sobre su nota final en la asignatura. Como muestra la Tabla 8 , se observa que más del $90 \%$ de los alumnos valoran bastante o muy positiva la repercusión en su calificación final.

En relación a la valoración de la influencia que hayan podido tener las actividades desarrolladas en las visitas guiadas a las empresas, en la mejora de ciertas capacidades personales de los alumnos, tomando como referencia el porcentaje de alumnos que les otorgan la valoración de bastante o muy positiva, destaca nuevamente el acercamiento a la práctica empresarial de los temas abordados en sus trabajos $(88,9 \%)$, seguida de la aplicación de nuevas tecnologías $(68,8)$ y del nivel de conocimiento alcanzado en dichas temáticas (66\%), como muestra la Tabla 9; por lo que se vuelve a confirmar el alto grado de consecución de los objetivos que nos habíamos propuesto con el desarrollo del Proyecto.

También en la encuesta realizada a los alumnos participantes durante el curso 2008-09, se ha añadido un nuevo ítem sobre el impacto que los alumnos han percibido en la influencia de la visita a la empresa como metodología docente innovadora. En este sentido, como muestra la Tabla 9, resulta muy relevante que el $82,3 \%$ de los alumnos valoran como bastante o muy positiva la innovación docente que ha representado la metodología aplicada en el desarrollo de las visitas a las empresas, otorgándole una puntuación media muy elevada, de 4,29 sobre 5.

\footnotetext{
${ }^{8}$ Vuelve a llamar la atención el hecho de que, como muestra la tabla 7 , sólo el $45,62 \%$ de los alumnos consideraba bastante o muy importante el objetivo de profundizar en el conocimiento de los temas abordados en clase, a la hora de participar en las prácticas voluntarias, y, sin embargo, cerca del $70 \%$ ha estimado que la influencia de su participación en las prácticas voluntarias sobre la adquisición de conocimientos ha sido bastante o muy positiva.
} 
TABLA 9. INFLUENCIA DE LAS VISITAS A LAS EMPRESAS EN LAS HABILIDADES PERSONALES DE LOS ALUMNOS Y EN LA INNOVACIÓN EN METODOLOGÍA DOCENTE. CURSO 08-09

\begin{tabular}{|c|c|c|c|c|c|c|c|c|c|c|}
\hline & \multicolumn{2}{|c|}{ Poco positiva } & \multicolumn{2}{|c|}{ Positiva } & \multicolumn{2}{|c|}{$\begin{array}{l}\text { Bastante } \\
\text { positiva }\end{array}$} & \multicolumn{2}{|c|}{ Muy positiva } & \multicolumn{2}{|c|}{ Total } \\
\hline & Frec. & $\%$ & Frec. & $\%$ & Frec & $\%$ & Frec & $\%$ & Frec & $\%$ \\
\hline $\begin{array}{l}\text { Acercamiento a la práctica } \\
\text { empresarial en los temas } \\
\text { tratados en la asignatura }\end{array}$ & 1 & 2,22 & 4 & 8,89 & 23 & 51,11 & 17 & 37,78 & 45 & 100 \\
\hline $\begin{array}{l}\text { Nivel de conocimiento de los } \\
\text { temas abordados }\end{array}$ & 1 & 2,22 & 14 & 31,11 & 21 & 46,67 & 9 & 20,00 & 45 & 100 \\
\hline Capacidad de comunicación & 1 & 2,22 & 14 & 31,11 & 17 & 37,78 & 13 & 28,89 & 45 & 100 \\
\hline $\begin{array}{c}\text { Aplicación de nuevas } \\
\text { tecnologías }\end{array}$ & 1 & 2,22 & 13 & 28,89 & 20 & 44,44 & 11 & 24,44 & 45 & 100 \\
\hline $\begin{array}{c}\text { Innovación en metodología } \\
\text { docente }\end{array}$ & & & 8 & 17,78 & 16 & 35,56 & 21 & 46,67 & 45 & 100 \\
\hline
\end{tabular}

En relación a la valoración global que los alumnos conceden a la utilidad de las prácticas, el 75,4\% de los alumnos participantes, como muestra la Tabla 10, le otorga la calificación de bastante o muy útil, situándose la valoración media en 3,89 sobre 5 puntos.

TABLA 10. VALORACIÓN GLOBAL DE LA UTILIDAD Y DE LA INFLUENCIA EN LA MEJORA DE LA DOCENCIA. CURSO 08-09

\begin{tabular}{|c|c|c|c|c|c|c|c|c|c|c|c|c|}
\hline & \multicolumn{2}{|c|}{ Nada útil } & \multicolumn{2}{|c|}{ Poco útil } & \multicolumn{2}{|c|}{$\begin{array}{l}\text { Nivel medio } \\
\text { de utilidad }\end{array}$} & \multicolumn{2}{|c|}{ Bastante útil } & \multicolumn{2}{|c|}{ Muy útil } & \multicolumn{2}{|c|}{ Total } \\
\hline & Frec. & $\%$ & Frec. & $\%$ & Frec. & $\%$ & Frec. & $\%$ & Frec. & $\%$ & Frec. & $\%$ \\
\hline $\begin{array}{l}\text { Valoración de la utilidad global de las } \\
\text { prácticas voluntarias }\end{array}$ & & & 2 & 3,51 & 12 & 21,05 & 33 & 57,89 & 10 & 17,54 & 57 & 100 \\
\hline Influencia en la mejora de la docencia & 1 & 1,75 & 1 & 1,75 & 13 & 22,81 & 29 & 50,88 & 13 & 22,81 & 57 & 100 \\
\hline
\end{tabular}

Finalmente, a través de otro nuevo ítem incorporado en la encuesta relativa al curso 08-09, y tal como recoge la Tabla 10, el 73,7\% de los alumnos otorgan la calificación de bastante o muy útil la influencia que el desarrollo de las prácticas ha tenido en la mejora de la docencia de la asignatura, con una valoración media de 3,91 sobre 5 puntos.

Como puede apreciarse, los resultados de las encuestas ponen de manifiesto que los objetivos que nos habíamos planteado con el Proyecto de Innovación Docente han sido alcanzados con éxito, avalado no sólo por la satisfacción del profesorado participante, sino también por los alumnos afectados.

\section{RESULTADOS}

Los resultados obtenidos a través del desarrollo del Proyecto de Innovación Docente se pueden diferenciar en dos tipos: tangibles e intangibles.

Entre los intangibles, destacan la contribución al desarrollo de ciertas habilidades personales en los alumnos participantes:

a) Habilidades de comunicación, mediante las reuniones con el tutor, las charlascoloquio en las empresas visitadas con los directivos de las mismas, la presentación pública del trabajo y el debate crítico dirigido por el tutor en clase.

b) Habilidades interpersonales, a través de la integración en grupos trabajo, la resolución de problemas dentro del grupo, la organización y delegación de tareas y la asunción de posiciones de liderazgo 
c) Destrezas con las tecnologías de la información y comunicación, mediante el uso de programas informáticos para la elaboración y presentación pública del trabajo, así como la utilización de bases de datos electrónicas especializadas.

También, dentro de los resultados intangibles, el profesorado participante ha tenido la oportunidad de acercarse a los problemas concretos que se presentan en las empresas visitadas, y establecer vínculos directos con el mundo de la empresa, lo que sin duda tendrá repercusiones muy positivas para el desarrollo futuro de la docencia y la investigación.

Entre los resultados tangibles destaca la mejora en el rendimiento académico de los alumnos. En efecto, como muestra la Tabla 11, durante los cursos en los que se han desarrollado los Proyectos de Innovación Docente?, se observa una mejora en la calificación media, que pasa de situarse entre el 3,81 y el 4,92 puntos sobre 10, a superar en todos los casos el aprobado, situándose entre 5,21 y 5,72 puntos. Además, el porcentaje de aprobados sobre los alumnos presentados se incrementa de forma notable, situándose entre el $66 \%$ y el $74 \%$, y el porcentaje de alumnos que obtienen una calificación igual o superior a 7 puntos sobre 10 también aumenta de forma considerable, alcanzando porcentajes entre el $22 \%$ y el $36 \%$ en el período referido.

TABLA 11. CALIFICACIONES CURSOS 2004-2010

\begin{tabular}{|l|c|c|c|c|c|c|c|}
\hline & feb-04 & feb-05 & feb-06 & feb-07 & feb-08 & feb-09 & feb-10 \\
\hline Alumnos matriculados & 183 & 173 & 116 & 117 & 149 & 138 & 157 \\
\hline Alumnos no presentados & 95 & 66 & 5 & 6 & 70 & 47 & 79 \\
\hline$\%$ alumnos no presentados & $52 \%$ & $38 \%$ & $4 \%$ & $5 \%$ & $47 \%$ & $34 \%$ & $50 \%$ \\
\hline Calificación media & 3,85 & 4,67 & 4,92 & $\mathbf{5 , 2 1}$ & $\mathbf{5 , 6 7}$ & $\mathbf{5 , 7 2}$ & 3,81 \\
\hline Alumnos aprobados & 34 & 62 & 69 & 73 & 58 & 67 & 31 \\
\hline$\%$ aprobados sobre matriculados & $19 \%$ & $36 \%$ & $59 \%$ & $62 \%$ & $39 \%$ & $49 \%$ & $20 \%$ \\
\hline$\%$ aprobados sobre presentados & $39 \%$ & $58 \%$ & $62 \%$ & $\mathbf{6 6} \%$ & $\mathbf{7 3 \%}$ & $\mathbf{7 4 \%}$ & $34 \%$ \\
\hline Alumnos con calificación 7-8 & 12 & 15 & 27 & 17 & 22 & 17 & 0,00 \\
\hline$\%$ calificaciones 7-8 sobre presentados & $14 \%$ & $14 \%$ & $24 \%$ & $\mathbf{1 5 \%}$ & $\mathbf{2 8 \%}$ & $\mathbf{1 9 \%}$ & 0,00 \\
\hline Alumnos con calificación 9-10 & 1 & 0,00 & 0,00 & 8 & 6 & 9 & 3 \\
\hline$\%$ calificaciones 9-10 sobre presentados & $1 \%$ & 0,00 & 0,00 & $\mathbf{7 \%}$ & $\mathbf{8 \%}$ & $\mathbf{1 0 \%}$ & $\mathbf{4} \%$ \\
\hline
\end{tabular}

Por último, también entre los resultados tangibles, el material didáctico audiovisual elaborado a partir de las visitas a las empresas será de gran utilidad para la docencia de las asignaturas afectadas en los cursos futuros.

\section{CONCLUSIONES}

La interrelación entre la teoría y la práctica profesional en el ámbito de la Contabilidad de Gestión resulta necesaria y conveniente. Mediante el desarrollo del Proyecto de Innovación Docente que, bajo el título de Docencia y Práctica Empresarial en Contabilidad de Gestión, hemos presentado en el presente trabajo, hemos pretendido establecer un puente de enlace entre la docencia de dicha asignatura y la práctica empresarial.

Entre los objetivos que nos habíamos propuesto con el desarrollo de dicho Proyecto resultan destacables el fomento de la interrelación entre profesores, alumnos y profesionales; la mejora del conocimiento de profesores y alumnos mediante la aproximación a la utilidad práctica de los temas abordados en clase; el logro de un acercamiento entre los problemas que se producen en la práctica contable

\footnotetext{
92007,2008 y 2009.
} 
empresarial y las soluciones que se proponen desde la teoría contable; la contribución al desarrollo de ciertas habilidades personales e interpersonales en los alumnos y de su destreza con las tecnologías de la información y comunicación; y el acceso de los alumnos a la investigación.

El análisis de las encuestas realizadas a los alumnos participantes para evaluar su satisfacción ha puesto de manifiesto un elevado grado de consecución de los objetivos que nos habíamos propuesto.

En efecto, si bien los alumnos manifestaban que su principal interés para participar en el proyecto residía en la obtención de una mayor puntuación en la calificación final, se observa una influencia bastante o muy positiva en la percepción que tienen los estudiantes sobre el nivel de conocimiento adquirido en los temas tratados, así como en el acercamiento a la práctica empresarial de los contenidos abordados. Asimismo, el alumnado muestra una elevada satisfacción en relación a la influencia que su participación en el proyecto ha tenido sobre el desarrollo de ciertas habilidades de trabajo en equipo, en su capacidad de comunicación, en el trabajo autónomo, así como en su aprendizaje en la utilización de bases de datos bibliográficas electrónicas y la aplicación de nuevas tecnologías.

Asimismo, resulta destacable la satisfacción experimentada por los profesores que han colaborado en el desarrollo de los Proyectos de Innovación Docente, tanto por los conocimientos adquiridos en su contacto con las empresas visitadas, como por los resultados obtenidos en el trabajo realizado por los alumnos participantes, que ha quedado reflejado en su rendimiento académico.

En consecuencia, según se desprende de los resultados obtenidos, la experiencia ha sido enriquecedora tanto para los alumnos participantes como para los profesores colaboradores. Además, en los próximos cursos académicos, los alumnos podrán conocer la experiencia previa realizada, gracias al material, tanto escrito como audiovisual, elaborado.

No obstante, la limitación que presenta el elevado número de alumnos, matriculados actualmente en las asignaturas de Contabilidad de Costes y de Gestión, dificulta la aplicación de la metodología propuesta en el presente trabajo. Limitación ésta que esperamos pueda ser superada con la puesta en marcha de estas asignaturas en los nuevos grados, en los que esperamos que el número de alumnos matriculados sea significativamente inferior, de forma que sea posible la generalización de las prácticas voluntarias y las visitas a las empresas recogidas en nuestros Proyectos de Innovación Docente.

Por otra parte, si bien en nuestra experiencia la propuesta fue recibida con entusiasmo por la dirección de las empresas con las que contactamos, el establecimiento de relaciones con empresas, que se muestren dispuestas a colaborar en el desarrollo de este tipo de proyectos, podría ser una dificultad añadida. En este sentido, sería conveniente la implicación de los Departamentos y otras instituciones universitarias para la consecución de contratos estables, que posibilitasen la aplicación de la metodología propuesta, de forma continuada, a lo largo de los distintos cursos académicos.

Finalmente, sería interesante la aplicación de la metodología propuesta, de forma conjunta, con los profesores de otras asignaturas afines, incluso de áreas de conocimiento distintas a la contable, en las que se aborden los mismos temas desde otras perspectivas, lo que permitiría ofrecer al alumno una visión global de la gestión de la empresa desde un punto de vista interdisciplinar. 


\section{BIBLIOGRAFÍA}

Accounting Education Change Commission [1990]: Position Statement num. 1. Objetives of Education for Accountants. AAA-AECC. Sarasota.

American AcCounting Association. Committee on the Future Structure, Content, and SCOpe OF AcCounting Education (The Bedford COMmitTeE). [1986]: Future Accounting Education: Preparing for the Expanding Profession. Issues in Accounting Education, Spring, pp. 168-195.

American Institute of Certified Public accountants. Education ExeCutive Committee [1988]: Education requirements for entry into the accounting profession. AICPA, New York.

American Institute of Certified Public accountants. Education ExeCutive Committee [1992]: Academic Preparation to Become a Certified Public Accountat. AICPA, New York.

American Institute of Certified Public accountants. Education ExeCutive Committee [1999]: The AICPA Core Competency Framework for Entry into the Accounting Profession. AICPA, New York. www.aicpa.org/edu.

ARQUERO , J.L. Y JIMÉNEZ, S.M. [1999]: Influencia del estudio de casos en la mejora del aprendizaje, adquisición de capacidades no técnicas y motivación en Análisis Contable, Revista de Enseñanza Universitaria, núm. Extraordinario: 225-241.

ARQUERO, J.L. Y DONOSO, J.A. [1998]: El cambio educativo en Contabilidad. El debate pendiente en España: formar para alcanzar el perfil adecuado del contable. VIII Encuentro de Profesores Universitarios de Contabilidad. Alicante, Mayo.

ArthUR ANDERSEN \& CO. ET. Al. [1989]: Perspectives on Education: Capabilities for Succes in the Accounting Profession. (White Paper). New York.

BEAVER, W. [1987]: Accounting Education: Challenges and Opportunities, Accounting Horizons, Diciembre: 1-6.

BLANCO DOPICO, M.I. [2007]: Contabilidad de gestión. Tópicos y agenda de investigación, Revista de Estudios Politécnicos, vol. IV, num. 7: 41-88.

Blanco Dopico, M.I. y Aibar, B. [2001]: La saga fuga de la Contabilidad de gestión o los gozos y las sombras de su investigación en España. VII Congreso del Instituto Internacional de Costos y II Congreso de ACODI, León, 4-6 Julio.

Cable, R.J., Healy, P. \& MATHEW, E. [2009]: Teaching Future Management Accountants, Management Accounting Quaterly, vol. 10, num 4, Summer: 44-50.

ESCOBAR, B. Y LOBO, A. [2000]: Docencia, investigación y práctica de la Contabilidad de gestión. Partida Doble, Julio-Agosto: 58-65.

GARCíA BenAu, M.A. [1997]: Algunas consideraciones internacionales sobre la controversia entre teoría y práctica contable. Revista Española de Financiación y Contabilidad, núm. 90, Enero-Marzo: 263-279.

GARCÍA BenAU, M.A.; GANDíA, J.L. Y VICO, A. [1996]: Relación entre teoría y práctica contable: una análisis de la situación en España. (AECA, Madrid).

INTERNATIONAL FEDERATION OF ACCOUNTANTS [1994]: 2000 and beyond. A strategic framework for prequalification education for the accountancy profession in theyear 2000 an beyond. IFAC, New York.

InTERnAtIONAL FEDERATION Of ACCOUNTANTS [1996]: Prequalification education, assessment of professional competence and experience requeriments of professional accountants. IFAC, New York.

TAN, L. M.; FOWLER, M.B. \& HAWKES, L. [2004]: Management accounting curricula: striking a balance between the views of educators and practitioners, Accounting Education, num. 13 (1), March: 51-67.

TUA, J. [1992]: La investigación en Contabilidad, una reflexión personal, Técnica Contable: 585-608. 